\title{
An arrayed-window microfluidic device for observation of mixed nanoparticles with an X-ray free-electron laser
}

\author{
Yutaka Matsumoto ${ }^{1}$. Yoko Takeo ${ }^{2,3}$. Satoru Egawa ${ }^{4}$. Gota Yamaguchi ${ }^{1}$. Shunya Yokomae ${ }^{1} \cdot$ Masashi Takei $^{5}$. \\ Hirokatsu Yumoto ${ }^{3} \cdot$ Takahisa Koyama $^{3} \cdot$ Haruhiko Ohashi $^{3} \cdot$ Kensuke Tono $^{3,6} \cdot$ Makina Yabashi, $^{3,6}$. \\ Hidekazu Mimura' ${ }^{1}$ Takashi Kimura ${ }^{2,7}$
}

Received: 11 September 2021 / Accepted: 9 December 2021 / Published online: 24 January 2022

(c) The Author(s) 2021

\begin{abstract}
A microfluidic device was developed for coherent diffraction imaging using an X-ray free-electron laser (XFEL-CDI). Liquid samples, which are separately packed in reservoirs of the device, are mixed in a flow channel after an XFEL irradiation as a trigger. An XFEL-CDI experiment was conducted and images of gold nanoparticles in solution were obtained with a resolution of several nanometers from single-shot coherent diffraction patterns. We were able to capture the images of various structures of mixed nanoparticles in the flow channel, clearly demonstrating the function of the microfluidic device. The direct observation of structural changes in liquids will contribute to development of chemistry and biology.
\end{abstract}

\section{Introduction}

X-ray free-electron lasers (XFELs) are characterized by high peak brightness, short pulse duration, and high spatial coherence [1]. While X-rays from conventional sources provide damages to samples, a femtosecond XFEL pulse enables us to obtain damage-free single-shot diffraction pattern before sample is destroyed, which is called the "diffraction-beforedestruction" scheme [2]34. In particular, the high peak

Takashi Kimura

tkimura@issp.u-tokyo.ac.jp

1 Department of Precision Engineering, Graduate School of Engineering, The University of Tokyo, 7-3-1 Hongo, Bunkyo-ku, Tokyo 113-8656, Japan

2 Institute for Solid State Physics, The University of Tokyo, 5-1-5 Kashiwanoha, Kashiwa, Chiba 277-8581, Japan

3 Japan Synchrotron Radiation Research Institute, 1-1-1 Kouto, Sayo-cho, Sayo-gun, Hyogo 679-5198, Japan

4 RIKEN Center for Advanced Photonics, 2-1 Hirosawa, Wako, Saitama 351-0198, Japan

5 Research Institute for Electronic Science, Hokkaido University, Kita 21 Nishi 10, Kita-ku, Sapporo 001-0021, Japan

6 RIKEN, SPring-8 Center, 1-1-1 Kouto, Sayo-cho, Sayo-gun, Hyogo 679-5148, Japan

7 Japan Science and Technology Agency, 7 Gobancho, Chiyoda-ku, Tokyo 102-0076, Japan brightness and coherence of XFEL facilitates us to obtain coherent diffraction patterns to analyze even non-crystalline samples [5]. Single-shot coherent diffraction imaging with XFEL (XFEL-CDI) has been used for structural analyses of varieties of nanometer-sized samples [6] 7 8. XFEL-CDI is particularly suitable for structural analysis of hydrated samples with high radiation sensitivity. For example, wet nanomaterials [9] 10 and live bacteria [11] 12 have been observed with XFEL-CDI. These studies have revealed the natural internal structure of samples without freezing or drying. The method also has the potential to capture instantaneous states during structural changes in liquids, which contribute to new discoveries in biology, chemistry and materials science.

Here, we report a proof-of-principle work on the observation of the instantaneous states of nanometer-sized samples in liquids by combining the XFEL-CDI technique with a microfluidic device. Microfluidic devices, which are assemblies of microfluidic channels and reaction containers fabricated using microfabrication technology, have been used for applications in biotechnology and chemical engineering [13] 14. In this study, we developed a new device which has microfluidic channels with a number of irradiation windows to allow efficient measurement of coherent diffraction patterns of samples in solutions with XFEL pulses. The channel of the device has two reservoirs to hold liquids separately. The liquids in the reservoirs flow into the channel when one of the windows is broken by an XFEL shot. We observed 
various aggregated conditions of spherical and rod-shaped gold nanoparticles mixed in the channel.

\section{Method}

\subsection{Experiment system}

The experiment was performed at BL2 of SPring-8 Angstrom Compact Free Electron Laser (SACLA) [15] 16. As shown in Fig. 1, the device containing liquid samples was placed in an instrument called MAXIC-S (an upgraded version of Multiple Application X-ray Imaging Chamber [17]) (see Fig. 2). An XFEL single pulse with a photon energy of $4 \mathrm{keV}$ and a duration of less than 10 femtoseconds was focused on an area of approximately $100 \mathrm{~nm} \times 100 \mathrm{~nm}$. The distance between the detector and the sample is $0.32 \mathrm{~m}$. The multi-port charge-coupled device (MPCCD) detector [18] with eight sensor modules has a square-shaped imaging area with about $100 \mathrm{~mm}$ per side. The maximum scattering angle from a sample is $8.9^{\circ}$, which gives a theoretical resolution of $1 \mathrm{~nm}$ per pixel in the reconstructed image. The coherent diffraction pattern has a Fourier transform relationship with the difference of the electron densities between the sample and the surrounding solvent. The image of the sample in solution is reconstructed by phase retrieval calculations [19] 20.

The microfluidic device shown in Fig. 3 is a square chip $(23 \mathrm{~mm} \times 23 \mathrm{~mm} \times 600 \mu \mathrm{m})$ with ten straight channels. Each channel has a dimension of $17 \mathrm{~mm} \times 0.5 \mathrm{~mm} \times 12 \mu \mathrm{m}$, two reservoirs that contain micro-liter liquid samples, and 45 irradiation windows made of a 200 -nm-thick film of silicon nitride. The window is a parallelogram of about $130 \mu \mathrm{m} \times 200 \mu \mathrm{m}$. The interval of each window is $300 \mu \mathrm{m}$. The liquid sample is first injected to the reservoir and sealed with a polyimide tape. In this initial condition, the liquids stay in the reservoirs due to the surface tension of the liquids. The device is then mounted on the sample holder in the vacuum chamber of MAXIC-S.

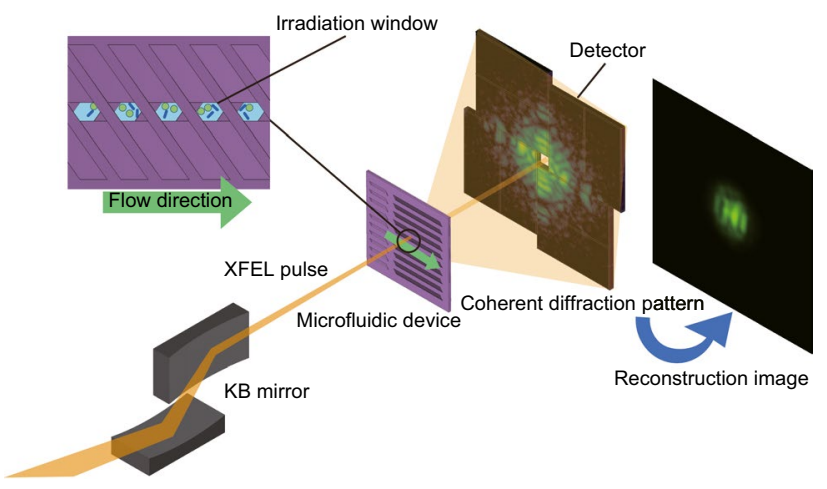

Fig. 1 Schematic illustration of the experimental system

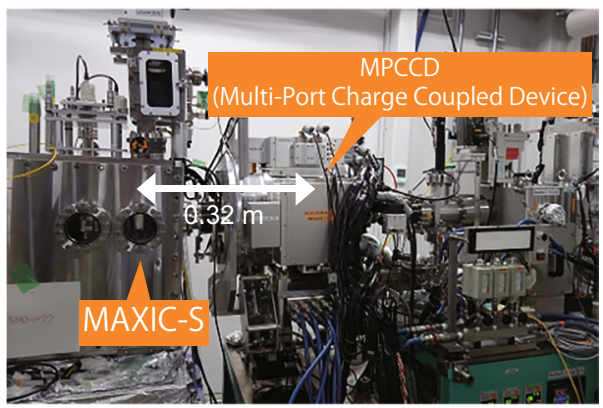

Fig. 2 XFEL-CDI measurement system at SACLA

An important feature of the microfluidic device is to allow observation of reactions of liquid samples immediately after they are mixed. Figure 4a shows a cross-sectional view of the microfluidic device with an XFEL beam path. With the first XFEL shot, a window at the end of the channel is broken, causing the liquid samples to flow into the channel, due to the rapid reduction of the pressure in the channel. Since each window is destroyed after single-shot irradiation, we need to change the windows for each irradiation. By irradiating the neighboring windows while shifting the device, several patterns from samples in solution can be obtained with the second and following shots. As shown in the Fig. 4b, c, the windows are irradiated from the window number 45 to 1 . By arranging snapshots of samples according to the window number, it is possible to capture structural changes of the samples in situ along the reaction time.

The flow velocity in the channels is estimated based on the following equation:

$v=\frac{\Delta p h^{2}}{\mu L}\left\{\frac{1}{12}-\frac{16 h}{\pi^{5} w} \tanh \left(\frac{\pi w}{2 h}\right)\right\}$,

where $v$ is flow velocity, $\Delta p$ is pressure, $\mu$ is fluid viscosity, $L$ is length of the channel, $h$ is height of the channel and $w$ is width of the channel [21]. In this experiment, $\Delta$ pis $1.0 \times$ $10^{5} \mathrm{~Pa}$ and $\mu$ is $9.4 \times 10^{-4} \mathrm{~kg} / \mathrm{m} \cdot \mathrm{s}$. $L, h$ and $w$ are $17 \mathrm{~mm}$,
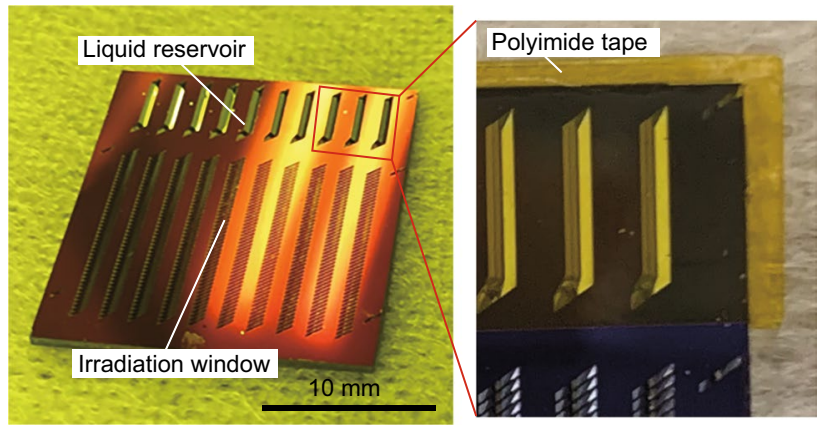

Fig. 3 Photograph of the microfluidic device and the polyimide tape on the device 
(a)

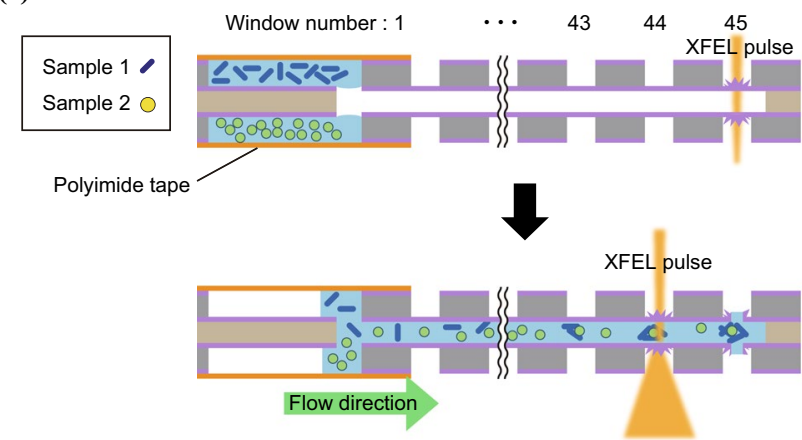

(b)

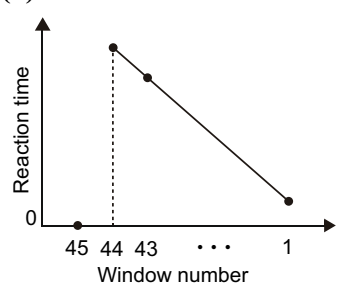

(c)

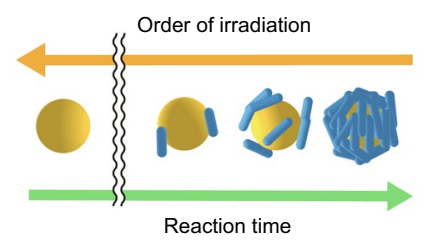

Fig. 4 a Cross-sectional illustration of the flow channel, b relationship between the window number and reaction time, c schematic illustration of structural development in the channel during the measurement

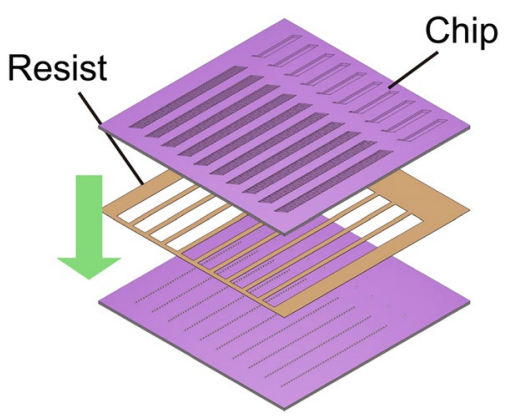

Fig. 5 Exploded view of the microfluidic device

$12 \mu \mathrm{m}$ and $0.50 \mathrm{~mm}$, respectively. The estimated flow velocity is $75 \mathrm{~mm} / \mathrm{s}$. Considering, this velocity and the interval of the windows, the time resolution of the obtained snapshots is $4.0 \mathrm{~ms}$.

\subsection{Fabrication of microfluidic device}

Figure 5 shows an exploded view of the microfluidic device. Two chips were fabricated by processing 4-inch silicon wafers with a thickness of $300 \mu \mathrm{m}$ and a plane orientation of (110). Since wet etching process proceeds in the direction perpendicular to the (110) surface of a Si wafer masked by the resist pattern with parallelogram shapes, the interval of the windows can be set to $300 \mu \mathrm{m}$, which improves the time resolution of the snapshots according to the Eq. (1) [22] 23.
Each silicon wafer had a 200-nm-thick silicon nitride layer on its surface. After spin-coating with the resist ZEP520A (ZEON Corp.) to a thickness of $500 \mathrm{~nm}$, the wafers were patterned by electron beam lithography (F5112 + VD01, Advantest). Reactive ion etching was subsequently used to remove the silicon nitride. In the area where the silicon was exposed, the irradiation windows and solution reservoirs were formed by wet etching with $30 \% \mathrm{KOH}$ aq. The patterned wafers were cut into $23 \mathrm{~mm}$ square chips by laser dicing (DFL7340, Disco). Finally, two chips were bonded together to make a single device. The patterned adhesive photoresist TMMR SA390N (Tokyo Ohka Kogyo Corp.) was used as the bond between the two chips and the side walls of the flow channels. The estimation of the actual flow velocity is difficult because photoresist thickness uniformity after spin coating was insufficient. The value of the height in the size of the channel has a range from 12 to $24 \mu \mathrm{m}$. According to the Eq. (1), the estimated time resolution also has a range from 4.0 to $1.0 \mathrm{~ms}$.

\subsection{Liquid samples}

In this study, gold nanoparticles (Cytodiagnostics Corp.) in two different shapes were used as samples in solution. One type was spherical gold nanoparticles and the other was rod-shaped ones. The gold nanospheres had a diameter of $50 \mathrm{~nm}$. The length and the diameter of a gold nanorod were $40 \mathrm{~nm}$ and $15 \mathrm{~nm}$, respectively. The nanospheres, which were covered with carboxyl groups, had a negative charge on their surface. The nanorod particles were covered with amino-PEG, having a positive charge on their surface. Due to the opposite charges, the two types of particles formed aggregates when the two solutions were mixed. Figures 6a, b show scanning electron microscope (SEM) images of a gold nanosphere and gold nanorods, respectively. Figure $6 \mathrm{c}$ shows an SEM image of an aggregate of gold nanoparticles.

Drops of the liquid samples were injected in the liquid reservoirs of both sides of the device with a pipette just before the XFEL-CDI experiment. The reservoirs were then sealed with polyimide tape, as shown in the right side of Fig. 3. UV-curing resin was applied to cover the edges of the tapes and to fill any small gaps.

\section{Results and discussions}

Figures $7 \mathrm{a}, \mathrm{b}$ show optical microscope images of the liquid reservoir of the device in the vacuum chamber, observed before and after XFEL irradiation. After the irradiation, the liquid sample was dissipated, indicating that the solution was triggered to flow into the channel by the XFEL pulse. Various types of coherent diffraction patterns were obtained by irradiating the samples in the channel. Figures $8 \mathrm{a}, \mathrm{b}, \mathrm{c}$ 
Fig. 6 SEM images of (a) a gold nanosphere particle, (b) gold nanorod particles, (c) an aggregate of gold nanorod particles and a gold nanosphere particle
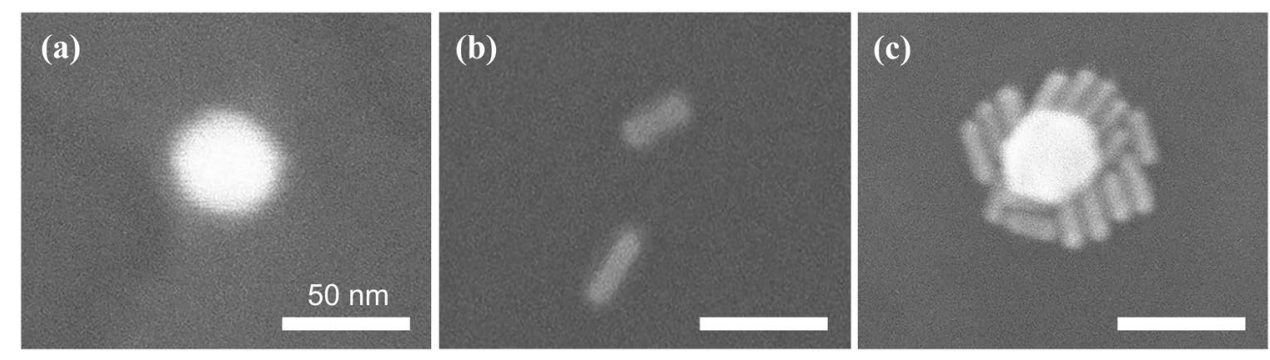
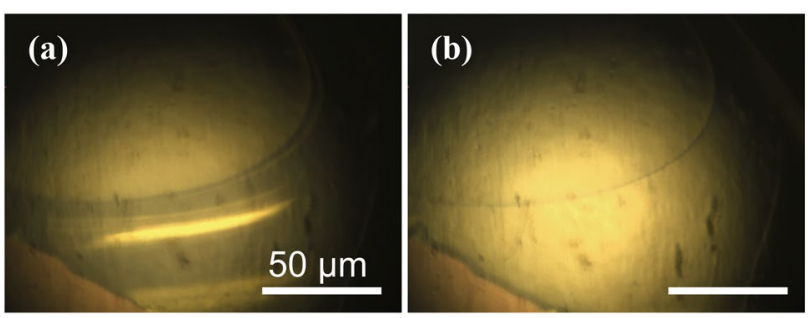

Fig. 7 Microscopic images of liquid reservoir (a) before and (b) after XFEL irradiation

show typical examples of the patterns. We used the relaxed averaged alternating reflections algorithm and the shrinkwarp algorithm [20] 24 to retrieve sample images from the coherent diffraction patterns. We performed 50 runs of image reconstructions from different initial random seeds for each diffraction pattern. Figures $8 d$, e, f show the averages of the 50 reconstruction results from the diffraction patterns shown in Fig. 8a, b, c, respectively. The images of the particles were retrieved with half-period spatial resolutions of $4.0 \mathrm{~nm}, 6.1 \mathrm{~nm}$, and $12.7 \mathrm{~nm}$, estimated by the phase retrieval transfer function [25]. This resolution allowed us to distinguish between gold nanosphere and nanorod particles. The values of phase shift contrast of 100-nm-thickness layers of $\mathrm{Au}$ and solvent are $3.7 \mathrm{rad} \times 10^{-1}$ and $3.0 \mathrm{rad} \times 10^{-2}$, respectively. Thus, the phase shift contrast loss of Au nanoparticles by solvent is almost negligible. The coherent diffraction pattern of a nanosphere particle was concentric, as shown in Fig. 8a. An intense streak in one direction appeared in the coherent diffraction pattern of a nanorod particle, as shown in Fig. 8b. We obtained several patterns caused by (a)

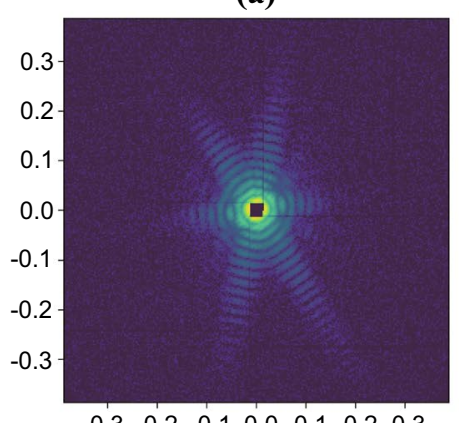

(d)

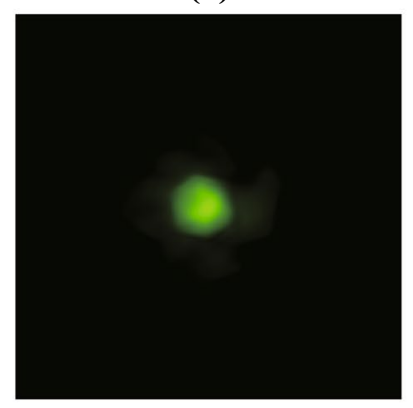

(b)

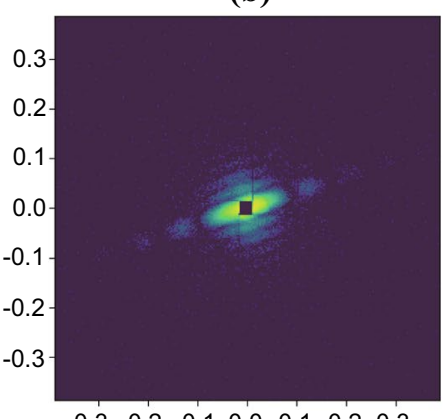

(e)

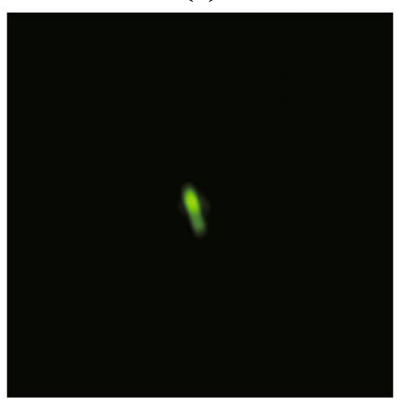

(c)

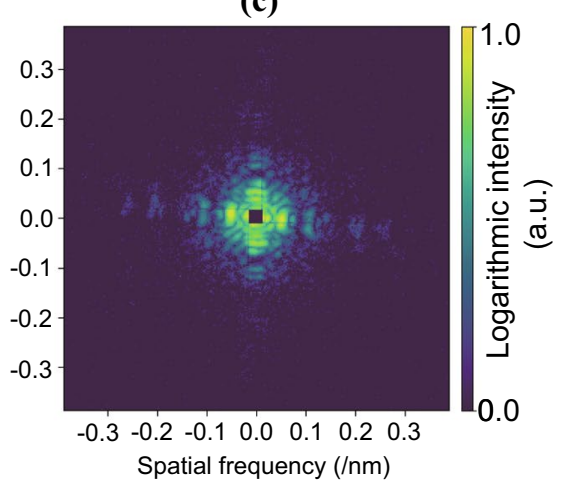

(f)

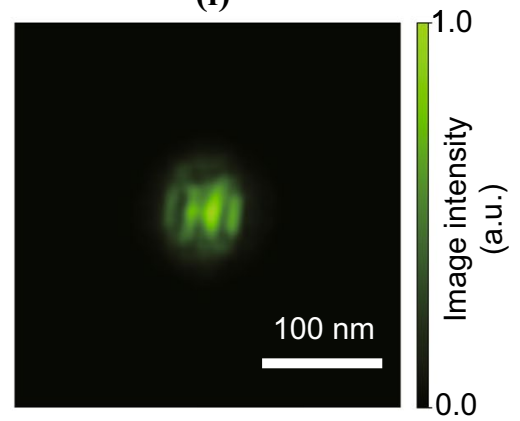

Fig. 8 Coherent diffraction patterns of (a) single gold nanosphere, (b) single gold nanorod, and (c) an aggregate of gold nanoparticles. d, e and $\mathbf{f}$ are the reconstructed images from $(\mathbf{a}),(\mathbf{b})$, and $(\mathbf{c})$, respectively 
nanorods, in which each intense streak was oriented in a random direction. One of the patterns is shown in Fig. 8b. We also obtained complex diffraction patterns that are different from these two types of patterns, as shown in Fig. 8c. In the image retrieved from Fig. 8c, an aggregate of many nanorod particles was observed. The image displays not only the three bright rod-shaped particles but also the dim-contrast objects at the top, left and bottom of the aggregate. We estimate that the center of the image looks brighter than the edges because of the Gaussian distribution of the illumination beam intensity. This image indicates that there was a nanosphere particle having a negative charge in the center of the aggregate. The nanorod particles were attached to the entire surface of the nanosphere particle. Aggregates of a nanosphere and nanorod particles were also observed with SEM of Fig. 6c. In both images of the aggregates shown in Figs. $8 \mathrm{f}$ and $6 \mathrm{c}$, multiple nanorod particles are attached to a nanosphere particle. In Fig. 8f, however, nanorod particles uniformly covers the surface of a nanosphere particle in contrast to the aggregate of nanorod particles lying on the membrane surface in Fig. 6c. We estimated that the electrostatic aggregate was broken due to the weak bonding of nanoparticles and the effect of drying process, such as the surface tension of the liquids and the concentration change. This result indicates that the method using the microfluidic device allows us to observe samples in solution without the effects of drying.

In this work, the device was designed to observe timeseries structural changes of nanometer-sized samples in solution. Although we obtained several images of particles in various aggregate forms, time-lapse observation of the aggregation process has not been achieved with these images. This is presumably due to the uncontrolled flow velocity and the insufficient amount of data. We need to optimize the concentration of the samples and the design of the device, for example, the cross-sectional area of the flow channel and the volume of the reservoirs.

\section{Conclusion}

We have developed a microfluidic device for XFEL-CDI experiments to observe nanometer-sized samples in solution. The device has microfluidic channels with two pairs of reservoirs and dozens of windows for irradiation with XFEL pulses for single-shot image acquisition. The first-pulse irradiation induces liquid samples held in the reservoirs to flow into the channels and start mixing. The experiments demonstrated the microfluidic device functioning properly, and several coherent diffraction patterns were acquired. Gold nanosphere particles, nanorod particles and aggregates of both were observed by phase retrieval calculations using the patterns. In the aggregates, nanorod particles were uniformly attached to the surface of a nanosphere particle. This state is different from that captured in SEM images. This indicates that the microfluidic device allows us to observe samples in their natural state. Our microfluidic device has the potential to enable time-lapse observations of the structural change process of nano-sized samples in liquid. Such observations will make an important contribution to the fields of chemistry and biology in the future.

Acknowledgements This work was supported by JSPS KAKENHI (Grant Number 20H04451 and 16H05989) and JST, PRESTO (Grant Number JPMJPR1772), Japan. The XFEL experiments were performed at the BL2 of SACLA (Proposal Numbers 2019B8050 and 2020A8044). This work was also supported by "Nanotechnology Platform Japan" of the Ministry of Education, Culture, Sports, Science and Technology (MEXT) Grant Number JPMXP09F20UT0148, and fabrication was conducted in Takeda Cleanroom with help of Nanofabrication Platform Center of School of Engineering, the University of Tokyo, Japan.

Open Access This article is licensed under a Creative Commons Attribution 4.0 International License, which permits use, sharing, adaptation, distribution and reproduction in any medium or format, as long as you give appropriate credit to the original author(s) and the source, provide a link to the Creative Commons licence, and indicate if changes were made. The images or other third party material in this article are included in the article's Creative Commons licence, unless indicated otherwise in a credit line to the material. If material is not included in the article's Creative Commons licence and your intended use is not permitted by statutory regulation or exceeds the permitted use, you will need to obtain permission directly from the copyright holder. To view a copy of this licence, visit http://creativecommons.org/licenses/by/4.0/.

\section{References}

1. Miao, J., et al.: Extending the methodology of X-ray crystallography to allow imaging of micrometre-sized non-crystalline specimens. Nat. 400, 342-344 (1999)

2. Neutze, R., et al.: Potential for biomolecular imaging with femtosecond X-ray pulses. Nat. 406, 752-757 (2000)

3. Bergh, M., et al.: Feasibility of imaging living cells at subnanometer resolutions by ultrafast X-ray diffraction. Q. Rev Biophys. 41, 181-204 (2008)

4. Chapman, H.N., et al.: Femtosecond diffractive imaging with a soft-X-ray free-electron laser. Nat Phys 2, 839-843 (2006)

5. Bogan, M., et al.: Single particle X-ray diffractive imaging. Nano Lett. 8, 310-316 (2007)

6. Chapman, H., et al.: Femtosecond X-ray protein nanocrystallography. Nat. 470, 73-77 (2011)

7. Ekeberg, T., et al.: Three-dimensional reconstruction of the giant mimivirus particle with an X-ray free-electron laser. Phys. Rev. Lett. 114(098102), 1-6 (2015)

8. Brändén, G., et al.: Coherent diffractive imaging of microtubules using an X-ray laser. Nat. Commun. 10, 2589 (2019)

9. Wojtas, D., et al.: Analysis of XFEL serial diffraction data from individual crystalline fibrils. IUCrJ. 4, 795-811 (2017)

10. Hunter, M., et al.: Fixed-target protein serial microcrystallography with an X-ray free electron laser. Sci. Rep. 4, 6026 (2014)

11. van der Schot, G., et al.: Imaging single cells in a beam of live cyanobacteria with an X-ray laser. Nat. Commun. 6, 5704 (2015) 
12. Kimura, T., et al.: Imaging live cell in micro-liquid enclosure by X-ray laser diffraction. Nat. Commun. 5, 3052 (2014)

13. Sarioglu, A., et al.: A microfluidic device for label-free, physical capture of circulating tumor cell clusters. Nat. Methods 12, 685-691 (2015)

14. Hung, L.H., et al.: Alternating droplet generation and controlled dynamic droplet fusion in microfluidic device for CdS nanoparticle synthesis. Lab. Chip 6, 174-178 (2006)

15. Ishikawa, T., et al.: A compact X-ray free-electron laser emitting in the sub-ångström region. Nat. Photonics 6, 540-544 (2012)

16. Tono, K., et al.: Multiple-beamline operation of SACLA. J. Synchrotron. Radiat. 26, 595-602 (2019)

17. Yumoto, H. et al.: High-fluence high-gain multilayer focusing optics to enhance spatial resolution in femtosecond $\mathrm{X}$-ray laser imaging. (submitted)

18. Kameshima, T. et al.: Development of an X-ray pixel detector with multi-port charge-coupled device for X-ray free-electron laser experiments. Rev. of scientific instruments 85, 033110 (2014)

19. Fienup, J.R.: Phase retrieval algorithms: a comparison. Appl. Opt. 21, 2758 (1982)

20. Marchesini, S. et al.: X-ray image reconstruction from a diffraction pattern alone. Phys. Rev. B - Condens. Matter Mater. Phys. 68, 1-4 (2003).
21. Bahrami, M., et al.: Pressure drop of fully-developed, laminar flow in microchannels of arbitrary cross-secction. ASME. 128, 1036-1044 (2006)

22. Prem Pal. et al.: A new model for the etching characteristics of corners formed by $\mathrm{Si}\{111\}$ planes on $\mathrm{Si}\{110\}$ wafer surface. Sci. Res. 5, 1-8 (2013)

23. Singh SS et al.: Determination of precise crystallographic directions for mask alignment in wet bulk micromachining for MEMS. Micro and Nano Sys. Let. 4, article number: 5 (2016)

24. Russell Luke D. et al. (2005) Relaxed averaged alternating reflections for diffraction imaging. Inverse Problem 21:37-50

25. Chapman, H.N., et al.: High-resolution ab initio three-dimensional X-ray diffraction microscopy. J. Opt. Soc. Am. A 23, 1179-1200 (2006)

Publisher's Note Springer Nature remains neutral with regard to jurisdictional claims in published maps and institutional affiliations. 